\title{
Uma Visão de Arquitetura para Redes 6G
}

\author{
Heitor Scalco Neto ${ }^{1,2}$, Antonia Vanessa D. Araujo ${ }^{2}$, Cristiano B. Both ${ }^{3}$, \\ Antonio Oliveira-Jr ${ }^{2,4}$, Kleber V. Cardoso ${ }^{2}$ \\ ${ }^{1}$ Instituto Federal de Mato Grosso do Sul (IFMS), Corumbá, MS, Brasil \\ ${ }^{2}$ Instituto de Informática - Universidade Federal de Goiás (UFG), GO, Brasil \\ ${ }^{3}$ Universidade Vale dos Sinos, São Leopoldo, RS, Brasil \\ ${ }^{4}$ Fraunhofer Portugal AICOS, Porto, Portugal \\ heitor.scalco@ifms.edu.br,antoniavanessa@discente.ufg.br \\ cbboth@unisinos.br, \{antoniojr,kleber\}@ufg.br
}

\begin{abstract}
The post-pandemic world must offer several opportunities for evolution in the network communications, as well as changing user experiences with augmented reality, connected industries, smart grids and self-organizing networks. This work presents the visions for new sixth generation (6G) network architectures, with the objective of discussing the key points that a new network architecture must encompass to promote the interaction of man with the world, whether physical and/or digital through a converged, flexible, intelligent and wide-ranging network for communication and computing.
\end{abstract}

Resumo. O mundo pós-pandemia deve oferecer diversas oportunidades de evolução nos meios de comunicação, bem como a mudança de experiências dos usuários com realidade aumentada, indústrias conectadas, redes inteligentes e redes auto-organizáveis. Este trabalho apresenta as visões para as novas arquiteturas de redes de sexta geração (6G), com o objetivo de discutir os pontos chave que uma nova arquitetura de rede deve englobar para promover a interação do homem com o mundo, seja ele físico elou digital por meio de uma rede convergente quanto a comunicação e computação, flexivel, inteligente e de ampla cobertura.

\section{Introdução}

O exponencial crescimento do número de aplicações e dispositivos que dependem de troca de tráfego massiva com outros dispositivos ou cloud (nuvem), assim como aplicações que requerem interação em tempo real, dispositivos que trabalham com inteligência artificial e automação, encontram algumas limitações nas tecnologias de redes móveis atuais, como a 5G. As redes de sexta geração, ou redes $6 \mathrm{G}$, devem atender a necessidade de evolução dos Key Performance Indicators (KPI) e expectativas não atendidas pela geração atual.

Como principais aspirações para as novas arquiteturas, pode-se citar a abrangência dos temas de enhanced Mobile Broadband (eMBB), massive Machine Type Communications (mMTC) e Ultra Reliable Low Latency Communications (URLLC), impulsionados pela otimização dos fundamentos tecnológicos de cloudization, deep slicing, softwarização e virtualização com ênfase na inteligentização da rede. Outro aspecto importante é a capacidade de deep slicing para garantir ampla conectividade e disponibilidade dos serviços por meio de coberturas de rede terrestres e não-terrestres [Samsung Electronics Co. 2021, Zhang et al. 2019, Mahmood et al. 2019]. 
Neste trabalho, parte-se da premissa de que as pesquisas em redes $6 \mathrm{G}$ já iniciaram, mesmo considerando a fase de implantação das redes 5G, inclusive no Brasil. A partir de uma revisão bibliográfica, apresentam-se as visões para o design arquitetônico de redes móveis 6G. Esse design está embasado na evolução das características da rede 5G, sobretudo na integração das arquiteturas Service-Based Architecture (SBA), baseada em micro serviços independentes e End-to-End Mandate-Driven Architecture (MDA), com "mandate" de Quality of Service (QoS) End-to-End (E2E), agnóstica de tecnologia e sistema operacional [Taleb 2020]. Com uma abordagem nativa de Inteligência Artificial (IA), as novas arquiteturas para 6G baseiam-se em rádios inteligentes e separação de Data plane (plano de dados) e User Plane (plano de usuário) para lidar com os recursos de hardware heterogêneos e atualizáveis, essa flexibilidade possibilita que as sub-redes locais evoluam e se atualizem individualmente [Letaief et al. 2019].

\section{Visões para a Arquitetura 6G}

A 3rd Generation Partnership Project (3GPP) especificou para a rede $5 \mathrm{G}$ a divisão das entidades de rede em duas grandes áreas: Rede de acesso por rádio, conhecida como $R a$ dio Access Network (RAN) e Núcleo (Core). Com uma arquitetura baseada em serviços, denominada Service-Based Architecture (SBA), as partes, RAN e Core, podem ser implementadas separadamente, sem dependências e com interoperabilidade.

Para atender as aspirações citadas, as arquiteturas de redes $6 \mathrm{G}$ devem promover a integração da arquitetura SBA baseada em micro serviços e da End-to-End MandateDriven Architecture (MDA), na qual os locatários e usuários finais se tornarão participantes ativos da arquitetura [Taleb 2020]. Uma SBA de próxima geração deverá promover no Core a decomposição dos serviços de rede em micro serviços independentes, enquanto na RAN, considerando a separação do plano de usuário da camada física, deve abordar um design de funções modularizadas, orientadas a serviços e divisão funcional [Taleb 2020].

A arquitetura MDA será conduzida por interações E2E entre os dispositivos finais, considerando todos os modelos de interação possíveis entre usuários e máquinas. Nesta abordagem cada "mandate" é visto como uma coleção de serviços de rede exigidos das redes subjacentes para atingir a qualidade de serviço necessária, ou seja, estratégias escalonáveis de monitoramento e verificação End-to-end $(E 2 E)$, implantadas em toda a rede, desde a parte de infraestrutura até os dispositivos finais onde os aplicativos estão em execução [Taleb 2020].

Além da integração SBA e MDA, conforme apresentado na Tabela 1, arquiteturas 6G devem apresentar o objetivo principal dos serviços, das características de rede e dos tipos de dispositivos que mais terão impacto na evolução da arquitetura 5G para a arquitetura 6G. A agregação de dispositivos e habilitadores tecnológicos, e.g. cloudization, deep slicing, softwarização, virtualização e inteligentização da rede; sensores e Distributed Ledger Technologies (DLT), Wireless Brain-Computer Interactions (BCI), vestíveis e implantes inteligentes, Extended Reality $(X R)^{1}$ ou Connected Robotics and Autonomous Systems (CRAS), representado por sistemas de entrega de drones, carros autônomos, enxames de drones autônomos e robótica autônoma ou ainda,[Saad et al. 2020], devem embasar a criação de novos cenários e casos de usos.

\footnotetext{
${ }^{1}$ Um novo termo que combina Virtual Reality (VR), Augmented Reality (AR) e Mix Reality (MR)
} 


\begin{tabular}{|c|l|l|}
\cline { 2 - 3 } \multicolumn{1}{c|}{} & \multicolumn{1}{c|}{ Arquitetura 5G } & \multicolumn{1}{c|}{ Arquitetura 6G } \\
\hline Objetivo & Conexão de Pessoas e Coisas & Interação de Pessoas e o Mundo \\
\hline \multirow{5}{*}{ Características } & Cloudization; & Inteligentização; \\
& Softwarização; & Cloudization; \\
& Virtualização; & Softwarização; \\
& Slicing. & Virtualização; \\
& & Deep Slicing. \\
\hline \multirow{5}{*}{ Dispositivos } & Smartphones; & Sensores e DLT; \\
& Sensores; & CRAS; \\
& Drones. & Equipamento XR e BCI; \\
& & Smart Implants. \\
\hline
\end{tabular}

Tabela 1. Aspectos evolutivos: Arquitetura 5G x Arquitetura 6G

\subsection{Tecnologias Habilitadoras}

De acordo com [Ziegler et al. 2020], os norteadores dos projetos para funções 6G incluem habilitadores tecnológicos que visam a simplificação, escalabilidade, flexibilidade, timeto-market ${ }^{2}$ e redução de erros. Outra característica fundamental de propostas de arquiteturas $6 \mathrm{G}$ é manter a diferenciação, assim como no $5 \mathrm{G}$, entre o control plane $(C P)$ e o user plane (UP), onde o $\mathrm{CP}$ será configurado como uma cadeia de serviços. Para atender os norteadores tecnológicos de simplificação das arquiteturas e funcionalidades, bem como de expansão da cobertura e inteligentização da rede, a literatura propõe um design que promova a convergência das RANs com o Core [Samsung Electronics Co. 2021], assunto que será discutido na próxima sub-seção.

\subsubsection{Convergência da RAN e Core}

Uma visão mais ampla sobre o significado da convergência entre RAN e Core é apresentada na Figura 1. Diferentemente da arquitetura proposta para redes 4G, em redes 5G, pode-se disponibilizar poder computacional (Core) nas estações rádio base, reduzindo drasticamente a latência das comunicações com a inclusão de dispositivos de borda. Para as arquiteturas $6 \mathrm{G}$ a visão é que o Core, ou seja, o poder de processamento estará presente tanto na RAN quanto nos equipamentos de usuários (UEs - User Equipments).

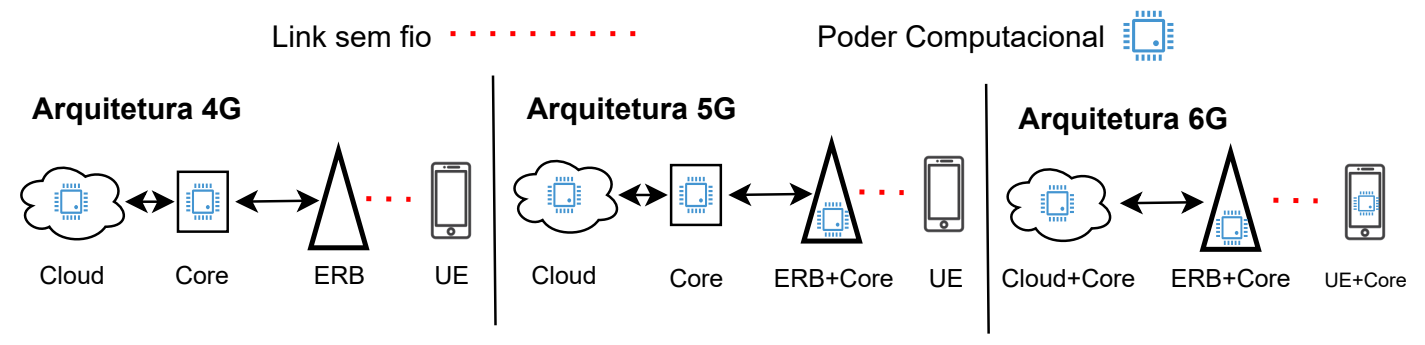

Figura 1. Evolução das Arquiteturas até a Convergência de RAN e Core

Uma das propostas para as redes $6 \mathrm{G}$ é a implementação de uma entidade chamada LLF (Lower Layer Function), que será responsável por incluir funções relacionadas a RAN que possuem requisitos críticos de latência, tanto no User Plane Micro Services (UPMS) quanto no Control Plane Micro Services (CPMS). Ao trazer essas funções para próximo da borda, alcança-se latências menores.

\footnotetext{
${ }^{2}$ Quantidade de tempo necessário para projetar e fabricar um produto até sua disponibilidade para venda.
} 
A Figura 2 apresenta um modelo de convergência entre RAN e Core, onde a LLF situada na borda pode conter características de cloud ou não. A LLF tem acesso a um micro serviço do plano de controle e plano de usuário. Por fim, tem-se a comunicação inter-cloud, especialmente no plano de usuário.

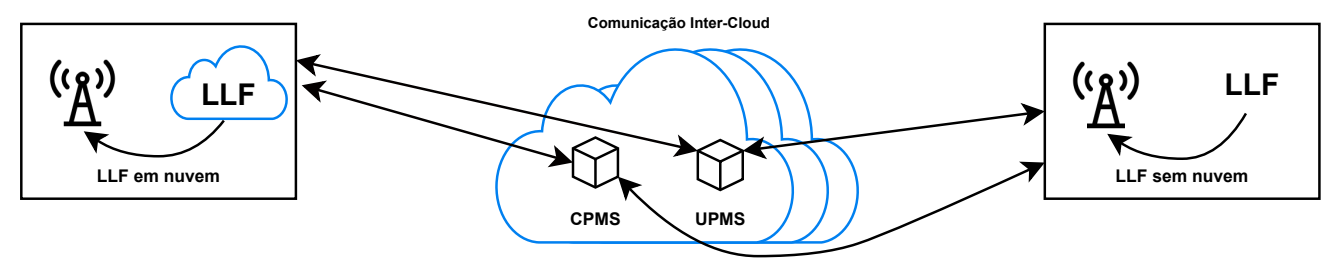

Figura 2. Convergência da RAN e Core

O CPMS inclui serviços de RAN e Core, tais como o controle de recursos do rádio, controle de rádio inteligente (Radio Intelligent Control $(R I C)$ ), gerenciamento de mobilidade, autenticação etc. Já o UPMS inclui os serviços das camadas mais altas da RAN no plano de usuário, bem como os serviços das camadas mais altas do Core, tais como: compressão de cabeçalho, criptografia, política de QoS e Deep Packet Inspection [Ziegler et al. 2020].

O UPMS e o CPMS serão baseados em uma estrutura que expõe APIs para novos micro serviços a serem adicionados ao conjunto principal de serviços que definem as duas entidades funcionais. Os micro serviços que constituem o UPMS e o CPMS podem ser alocados em uma nuvem híbrida de forma desagregada, onde pode haver uma instância local e uma central do UPMS atendendo a diferentes conjuntos de casos de uso.

A convergência de RAN e Core, incluindo as otimizações funcionais, permitirão o surgimento de RANs altamente especializadas (por exemplo: vehicle-to-vehicle communication $(V 2 V)$ e RANs para slices específicos da rede. A facilidade de introdução de novos serviços e novos tipos de dispositivos com diferentes recursos de rádio e pilhas de software dedicadas otimizará o time-to-market e o custo total.

\subsubsection{Conexões em Malha (Mesh)}

Outro fator importante para o desenho das arquiteturas $6 \mathrm{G}$ é a ausência da separação entre células para a conectividade por áreas. A conectividade dupla entre New-Radio e New-Radio (NR-NR), permite a formação de redes Mesh, na qual um dispositivo pode ser associado a uma "célula" principal e uma escrava, diferentemente do que ocorre nas redes 5G e anteriores, onde um dispositivo é associado a apenas uma célula. Dispositivos 6G podem conectar-se a diversos dispositivos próximos para formar sub-redes $6 \mathrm{G}$ enquanto mantém a conectividade com o nó principal. As arquiteturas $6 \mathrm{G}$ devem ser projetadas para nativamente suportar conectividade Mesh, o que deve ser possível a partir da alocação do UP e CP em nuvem, com a desassociação do modelo de conexão por células para facilitar a mobilidade entre as redes. 


\subsubsection{Inteligentização da rede}

O design 6G requer a mudança do clássico paradigma Self-Organizing Networks (SON), para o SSN Self-Sustaing Networks (SSN), ou seja, de redes auto-organizadas para redes autossustentáveis. As SSNs devem ser capazes de, além de adaptar suas funções, sustentar seu uso e gerenciamento de recursos (ex.: captando energia e explorando espectro) para manter de forma autônoma KPIs altos e de longo prazo. Para atender os requisitos de automação previstos, as funções das SSNs devem aproveitar as recentes evoluções nas tecnologias de IA [Saad et al. 2020] que, considerando as entidades de redes, podem ser implementadas e categorizadas em três níveis: IA local, IA Conjunta, IA E2E [Samsung Electronics Co. 2021].

- IA local: implementada em cada entidade da rede, realiza tarefas como otimização da modulação, codificação da fonte e codificação do canal.

- IA Conjunta: implementada para otimizar a operação conjunta de UEs e BSs (Base Stations) ou a operação conjunta de redes centrais e servidores de aplicativos, como a adaptação com base na previsão das condições futuras da rede em ambientes sem fio complexos.

- IA E2E: implementa em todo o sistema de comunicação, possibilitando identificar ou prever anomalias no funcionamento da rede e sugerir ações corretivas.

\subsubsection{Expansão da Cobertura}

No intuito de garantir ampla cobertura, as arquiteturas de rede 6G devem ser projetadas para integrar as terrestrial networks (TN) com as entidades non-terrestrial networks (NTN) como: aviões, urban air mobility systems (UAM), satélites low earth orbit (LEO) e geo-stationary orbit (GEO), e high altitude platform stations (HAPS).

As topologias de redes futuras devem evoluir, sobretudo com o uso dos componentes NTN, tais como: satélites e HAPS, para garantir coberturas em áreas onde não há rede terrestre. Esta abordagem ainda apresenta muitos desafios de pesquisa, sobre aspectos conhecidos, porém ausentes nas redes terrestres e aspectos adicionais, ainda não reconhecidos, que podem surgir e precisam ser considerados ao implementar redes com suporte a NTN [Samsung Electronics Co. 2021].

\section{Discussões, Direcionamento e Conclusões}

Os modelos de arquitetura propostos para as redes 6G ainda são abstratos para uma implementação inicial. Entretanto, já é possível, embasados na evolução dos cenários eMBB, URLLC e mMTC propostos para redes 6G, visualizar e discutir a evolução das arquiteturas com foco na hiper conectividade e interações do homem com o mundo, seja digital ou físico, propostos na literatura. Além do objetivo dos serviços disponibilizados, as características desses serviços, bem como as estruturas das redes, devem ser fortemente embasados nos fundamentos tecnológicos de cloudization, deep slicing, softwarização, virtualização e inteligentização da rede, sendo este último um dos aspectos de maior destaque na literatura.

Acredita-se que os projetos das arquiteturas $6 \mathrm{G}$ estarão embasados na evolução da arquitetura SBA, proposta para redes $5 \mathrm{G}$, em conjunto com a arquitetura MDA/E2E, com 
IA nativa. Essa integração deve prover micro serviços independentes, agrupados de forma inteligente em "mandates" que especifiquem e garantam QoS/E2E na rede. Outro aspecto importante é a convergência entre RAN e Core, que podem a partir de uma nuvem híbrida prover simplificação e conectividade em rede Mesh; além de proporcionar que casos de uso onde não há infraestrutura definida (por motivos de localização remota ou desastres) sejam explorados, e.g. conectividade em veículos de mineração, comunicação de viaturas de segurança pública, swarm de drones, redes de sensores e outros. A principal ideia da convergência de RAN e Core é fazer com que dispositivos possam criar suas próprias sub-redes e auto gerenciá-las.

A flexibilidade oferecida pelas arquiteturas $6 \mathrm{G}$ deve permitir um alto nível de especialização das redes para propósitos específicos, tais como: sub-redes e slices otimizados por tipo de tráfego. As arquiteturas dos componentes de orquestração baseadas em domínios, redes e automação serão grandes habilitadores para a implementação em indústrias. Alguns outros aspectos ainda não estão bem definidos nas propostas de arquiteturas $6 \mathrm{G}$, um deles é como serão tratadas questões de segurança, visto a utilização de micro serviços com alta flexibilidade, podendo ser alocados em nuvens ou UEs que podem ter recursos e funcionalidades variadas, rodando ao mesmo tempo. As escolhas de protocolos de rede e políticas de controle ainda não foram definidas e podem ser desenvolvidas em trabalhos futuros.

\section{Agradecimentos}

O presente trabalho foi realizado com apoio do Projeto Brasil 6G, financiado pelo Ministério da Ciência, Tecnologia e Inovações (MCTI) e Rede Nacional de Pesquisa (RNP).

\section{Referências}

Letaief, K. B., Chen, W., Shi, Y., Zhang, J., and Zhang, Y. A. (2019). The Roadmap to 6G: AI Empowered Wireless Networks. IEEE Communications Magazine, 57(8):8490. Conference Name: IEEE Communications Magazine.

Mahmood, N. H., Alves, H., López, O. A., Shehab, M., Osorio, D. P. M., and Latva-aho, M. (2019). Six key enablers for machine type communication in 6g. arXiv preprint arXiv:1903.05406.

Saad, W., Bennis, M., and Chen, M. (2020). A Vision of 6G Wireless Systems: Applications, Trends, Technologies, and Open Research Problems. IEEE Network, 34(3):134142. Conference Name: IEEE Network.

Samsung Electronics Co., L. (2021). 6G the next hyper Connected Experience for All | Samsung Research.

Taleb, T. (2020). White paper on 6G networking. Publisher: Oulu : University of Oulu,.

Zhang, Z., Xiao, Y., Ma, Z., Xiao, M., Ding, Z., Lei, X., Karagiannidis, G. K., and Fan, P. (2019). 6g wireless networks: Vision, requirements, architecture, and key technologies. IEEE Vehicular Technology Magazine, 14(3):28-41.

Ziegler, V., Viswanathan, H., Flinck, H., Hoffmann, M., Räisänen, V., and Hätönen, K. (2020). 6G Architecture to Connect the Worlds. IEEE Access, 8:173508-173520. 